\title{
Inteligencia emocional e intención emprendedora de los estudiantes en la Facultad de Ciencias Empresariales de la Universidad Peruana Unión, filiales Lima, Juliaca y Tarapoto, 2016-II Emotional intelligence and entrepreneurial intention of students in the Faculty of Business Sciences of the Universidad Peruana Unión, Lima, Juliaca and Tarapoto subsidiaries, 2016-II
}

\author{
Erlita Yamelin Chávez Paz ${ }^{1 *}$, Jenniffer Consuelo Suárez Quijano¹, Elizabe- \\ th García Salirrosas ${ }^{1 \star \star}$, Edison Effer Apaza Tarqui ${ }^{1 \star \star \star}$. \\ 'Escuela Profesional de Administración, Facultad de Ciencias Empresariales, Universidad Peruana Unión
}

INFORMACIÓN DEL ARTÍCULO

Historia del artículo

Recibido: 12 noviembre de 2016

Aceptado: 28 abril de 2017

\section{Resumen}

El objetivo de esta investigación fue determinar la relación entre la inteligencia emocional y la intención emprendedora de los estudiantes universitarios del sexto y octavo ciclo de la Facultad de Ciencias Empresariales en la Universidad Peruana Unión, con sede en Lima y sus filiales en Tarapoto y Juliaca, durante el ciclo 2016II. El estudio fue de tipo correlacional con diseño no experimental y de corte transversal. La muestra no probabilística estuvo constituida por 422 universitarios. Los instrumentos utilizados fueron el TMMS-24, que mide las dimensiones atención emocional, claridad emocional y reparación emocional, y el cuestionario Intención Emprendedora, compuesto por seis dimensiones: antecedentes personales y familiares, normas sociales, características emprendedoras, creencias, actitud y oportunidades y recursos. Para el análisis estadístico se utilizó tablas de frecuencia y porcentajes, para determinar los niveles de intención emprendedora e inteligencia emocional en los estudiantes. Según los resultados se demostró, mediante el coeficiente Rho de Sperman, una relación positiva, directa y significativa entre las variables y las dimensiones. Asimismo, los niveles de intención emprendedora en los estudiantes de la sede y sus dos filiales fue de $68.8 \%$, mientras que el de intención emprendedora fue de $70.2 \%$. También se encontró que en la filial de Tarapoto, los niveles de inteligencia emocional e intención emprendedora, son los más bajos, comparado con a la sede en Lima y la filial Juliaca. Finalmente se concluye que a mayor nivel de inteligencia emocional, los estudiantes unionistas poseen mayor intención de emprender.
Palabras clave:
Inteligencia emocional, intención emprendedora, atención, claridad, reparación.

\begin{abstract}
Abstrac
The objective of this research was to determine the relationship between emotional intelligence and the entrepreneurial intention of university students of the sixth and eighth cycles of the Faculty of Business Studies at Universidad Peruana Unión, based in Lima and its subsidiaries in Tarapoto and Juliaca, during the 2016-II cycle. The study was of a correlational type with a non-experimental and cross-sectional design. The non-probabilistic sample con-
\end{abstract}


Keywords:

Emotional intelligence, entrepreneurial intention, attention, clarity, repair.

\begin{abstract}
sisted of 422 university students. The instruments used were the TMMS-24, which measures the dimensions of emotional attention, emotional clarity and emotional reparation, and the questionnaire "Intent entrepreneur", composed of six dimensions: personal and family background, social norms, entrepreneurial characteristics, beliefs, attitude and opportunities and resources. For the statistical analysis, frequency tables and percentages were used to determine the levels of entrepreneurial intention and emotional intelligence in the students. According to the results, a positive, direct and significant relationship between the variables and the dimensions was demonstrated using the Rho coefficient of Sperman. Likewise, the levels of entrepreneurial intention in the students of the headquarters and its two subsidiaries was $68.8 \%$, while the level of entrepreneurial intention was $70.2 \%$. It was also found that in the subsidiary of Tarapoto, the levels of emotional intelligence and entrepreneurial intention are the lowest, compared to the headquarters in Lima and the subsidiary Juliaca. Finally, it is concluded that at a higher level of emotional intelligence, unionist students have a greater intention to undertake.
\end{abstract}

\section{Introducción}

Hidalgo, Kamiya y Reyes (2014) mencionaron que la creación y crecimiento de empresas es una de las fuentes más importantes que generan riquezas y empleos en la población, por lo tanto es una potencial herramienta para reducir la pobreza y elevar los ingresos; es por esta razón que el emprendimiento dinámico se ha convertido en un tema de creciente interés en la política pública de los países de América Latina y del mundo.

A nivel mundial, Singer, Amorós y Moska (2014) informaron, en el GEM 2014, que las economías con mayores tasas de intenciones emprendedoras, son Uganda (60,2\%), Camerún (55,6\%), Qatar $(50,4 \%)$, Perú $(50,6 \%)$ y Chile $(50,1 \%)$.

Mientras que en Sudamérica, según Reátegui (2015), en el año 2014, Ecuador con un $32,6 \%$, fue líder en la región y considerado el cuarto a nivel mundial, siguiendo Perú con una tasa de actividad emprendedora de $28,8 \%$ superando a Bolivia $(27,4 \%)$ y Chile $(26,6 \%)$. Sin embargo, en el reporte del GEM 2015, el porcentaje de tasa de actividad empren- dedora en Perú disminuyó a un 22.2\%, ubicándose en el ranking número nueve de las sesenta economías evaluadas. (Kelley, Singer, y Herrington, 2016)

Villarán (2004) mencionó que países en proceso de desarrollo como el nuestro, enfrentan grandes retos como la generación de empleos de calidad, mejora de la competitividad y exportación. Zúñiga (2015) refirió que en muchos países como en el Perú, los emprendedores han encontrado en la actividad empresarial una forma de vida, teniendo a las pequeñas y medianas empresas (Pymes), como protagonistas fundamentales en su economía, disminuyendo la pobreza e incrementando el producto bruto interno del país. Según un estudio realizado por el Ministerio de la Producción, en el 2007, las microempresas ocupaban el 97.8\% mientras que las pequeñas empresas comprendían el $2.1 \%$, mientras que en el año 2014, la Asamblea de Emprendedores del Perú (ASEP), realizó una investigación, obteniendo resultados de un $87.8 \%$ y un $10.9 \%$, respectivamente. Por ello, la ASEP (2015) determinó que a las microempresas les cuesta avanzar al siguiente nivel, siendo como común de- 
nominador que mayormente estas retroceden hacia una microempresa, en lugar de avanzar hacia una mediana; esto es, según Salazar (2016), porque las empresas están enfocadas en sobrevivir y no pueden crecer y mucho menos innovar. Frente a este desafío, el gobierno ha implementado ciertas leyes que promueven un ambiente favorable para el ecosistema emprendedor: la Ley $N^{\circ} 28015$ (Ley de Promoción y Formalización de la Micro y Pequeña Empresa) aprobada el 03/07/2003. El capítulo 1, artículo 5 explica el papel del gobierno frente al ecosistema emprendedor, fomentando el espíritu emprendedor y creativo de la población, al promover la inversión privada y nacional en apoyo a las Mype, reforzado por el art. 14 que promueve la conformación de mercados de servicios financieros y no financieros, de calidad, descentralizado y pertinentes a las necesidades de la Mype. Asimismo, la Ley N 30056 (ley que modifica diversas leyes para facilitar la inversión, impulsar el desarrollo productivo y el crecimiento empresarial) promulgada el 02/07/2013; en el artículo 1 se especifica el objetivo de establecer el marco legal para la promoción de las Mype, con políticas de alcance general y la creación de instrumentos de apoyo y promoción, incentivando la inversión privada, la producción, el acceso a los mercados internos y externos y otras políticas que impulsen el emprendimiento y permitan la mejora y el crecimiento sostenido de estas unidades económicas. Asimismo, en el art. 13, inciso uno, describe su ayuda mediante el cofinanciamiento de emprendimientos dinámicos y de alto impacto, con un enfoque que los oriente hacia el desarrollo nacional, la internacionalización y la permanente innovación, dicho financiamiento se hará efectivo mediante el Ministerio de la Producción.

De la misma manera, la ley $N^{\circ} 30220$ (Ley Universitaria), aprobada el 03/07/2014 señala en el capítulo $\mathrm{VI}$, articulo 52, la responsabilidad de promover la iniciativa de los estudiantes para la creación de pequeñas y microempresas de propiedad de los estudiantes, brindando asesoría o facilidades en el uso de los equipos e instalaciones de la institución.

También, el gobierno viene promocionando diversos programas que ayudan a los emprendedores a lanzarse al mundo empresarial: Start Up, Cofide, Impulsa Perú, Innóvate Perú. Del mismo modo existen incubadoras a nivel nacional en instituciones privadas como: Asociación de Emprendedores del Perú, Kunan, Endeavor. Y por ultimo, está la red de incubadoras de las universidades a nivel nacional: Centro de Innovación y Desarrollo Emprendedor (Pontificia Universidad Católica), Universidad Nacional Mayor de San Marcos.

La Facultad de Ciencias Empresariales de la Universidad Peruana Unión, tiene como Misión y Visión "Formar profesionales e investigadores emprendedores, para el servicio a la sociedad". Al respecto desde el año 2002 hasta el año 2014 funcionó el INCUBEM - UPeU (Incubadora de empresas de la Universidad Peruana Unión), dentro de la Facultad de Ciencias Empresariales, después pasó al departamento de Dirección General de Investigación de la Universidad Peruana Unión. Actualmente en la malla curricular de la carrera de Administración, existen los cursos de creatividad y emprendedorismo que se dicta en tres niveles. Como parte del desarrollo de esta materia durante los dos últimos años (2015 y 2016), antes de finalizar las actividades académicas, se viene realizando la "Semana Global del Emprendimiento". A parte de estas actividades para promover el espíritu emprendedor en los estudiantes no se tiene evidencias de otras actividades con este propósito. 
Por otro lado, Acuña (2014) en su investigación realizada en la Universidad Peruana Unión filial Tarapoto, encontró que uno de los grandes problemas que afecta el ecosistema emprendedor, en la universidad, es el esquema de las asignaturas de emprendimiento dictadas, siendo solo teóricas y dejando de lado lo práctico, teniendo en cuenta que se require desarrollar habilidades para una actuación competente en el mercado laboral tan exigente.

Por todo ello, el presente trabajo cuestiona, si solo será necesario implementar un ecosistema para el emprendimiento cuyos factores son netamente externos para inspirar el espíritu emprendedor, o si será necesario conocer los factores internos que influyen en una persona para emprender. Por ende, tomando en cuenta los estudios realizados por Goleman, se sustentó que la inteligencia emocional es responsable de entre el $85 \%$ y el $90 \%$ del éxito de los líderes de las organizaciones (Diez, 2011). Por eso, Del Fabro (2013), en su trabajo de investigación se buscó encontrar una relación con la inteligencia emocional como factor imprescindible para emprender.

Asimismo, Pittaluga (2012) hace hincapié en que la inteligencia emocional debería ocupar una posición de privilegio en los programas formativos de las escuelas de gerencia. Ya que como mencionan Fernández y Extremera (2002), las organizaciones son determinadas en gran parte por la habilidad en inteligencia emocional de sus líderes.

\section{Revisión de la literatura}

\section{Emprendedorismo}

El emprendedurismo es definido como la capacidad de una persona para generar empresa (Puchol, 2005), innovar
(Hernández, Cabellero, y Monroy, 2013) y asumir riesgos (Bautista, 2014). Dando por hecho que la actividad generada por un emprendedor en un proyecto o negocio, es fundamental para el desarrollo social y económico del país (Numancia, 2013).

El emprendedorismo puede ser tratado como un plus adicional, como fuente de creación de negocios, y como un proceso que incentiva a la innovación. Celaya (2013) consideró al emprendedorismo como un plus adicional; este plus declara Silva (2013) citado por Bautista (2014), que es la asunción de riesgos, la cual permite aventurarse en la creación de negocios. Siendo este último, el resultado final de una incertidumbre considerable (Alvaro, Mendiri, y Paz, 2003).

Por otro lado, para Puchol (2005), el emprendedorismo es una fuente de creación de negocios que se le incentiva a una persona; concepto creado por Schumpeter quien aportó el término emprendedor para definir a estas personas (Nueno 2007). Es así que Morant Climent (2012) citado por Gómez, Mira, Martínez y Estrada (2012), definió al emprendedorismo, como una fuente de creación de riquezas por medio de la formación de negocios propios; considerando al emprendedor, como el motor para la realización de una empresa, superando las adversidades que esto supone (Alonso y Galve, 2008).

Finalmente, Fretes, Venturi, Ocampo, y Villalba (2016) en su tesis, describieron a la variable emprendedorismo como el proceso que incentiva a la innovación; concepto apoyado por Bolton y Thompson (2000), citado por Hernández, Cabellero, y Monroy (2013) quienes definen al emprendedor como una persona que habitualmente innova cosas partiendo de todo tipo de oportunidades y/o necesidades. 


\section{Inteligencia emocional}

La inteligencia emocional fue definida como la habilidad para percibir, comprender y usar las emociones adecuadamente (Fernández, Extremera y Ramos, 2003; Pittaluga, 2012; Salguero, Fernández, Ruiz, Castillo, y Palomera, 2015).

Denominada también como la capacidad para reconocer, entender y aplicar eficazmente las emociones, utilizándolas para solucionar problemas presentados en un entorno tan cambiante. (Ferrando, 2006; Cooper y Sawaf, 1998, citado por Quintillán, 2005; Valles, 2005, citado por Martin de Benito, 2013; Cabrera, 2011)

Y por último, la inteligencia emocional fue conceptualizada como un conjunto de competencias que determinan el comportamiento de un individuo, usados para afrontar diversas situaciones, considerándola indispensable para el éxito de las organizaciones (Abrego, González y Lullo, 2012; Manrique, 2012; Goleman, 1995, citado por Bollón, 2010; Martin de Benito, 2013; Maestres, 2006; Soto, De la Torre, y Piñon, 2014). En pocas palabras, la inteligencia emocional, es el uso inteligente de las emociones. (Weisinger, 1998, citado por Martín de Benito, 2013)

\section{Materiales y métodos}

El tipo de investigación es de alcance correlacional, y no experimental porque no se manipulará las variables de estudio durante la investigación. Asimismo es transversal porque se tomaron los datos en un solo momento.

\section{Participantes}

El número de estudiantes es de 604, los cuales pertenecen a la Facultad de Ciencias Empresariales y a las carreras de
Administración, Contabilidad y Marketing en sus respectivas filiales, Tarapoto, Juliaca y Lima. Los participantes estuvieron matriculados en el periodo 2016 - II.

\section{Criterios de inclusión}

Para la ejecución de esta investigación, se trabajó con ciertos criterios de inclusión, los cuales son mencionados a continuación:

- Estudiantes mayores de 18 años.

- Estudiantes matriculados en el sexto y octavo ciclo de las carreras de Administración, Contabilidad y Marketing, con sus respectivas especialidades.

- Estudiantes con carga regular presencial (lista brindada por los directores de escuela profesional, de cada filial)

- Estudiantes que tengan en su carga académica, los cursos de emprendedorismo asignados por su respectivo plan de estudio.

- Estudiantes con la voluntad de apoyar en el llenado de encuestas, para esta investigación.

Tabla 1

Districución de los participantes por ciclo y carreras

\begin{tabular}{llc}
\hline & & Frecuencia \\
\hline Ciclo & VI ciclo & 200 \\
& VIII ciclo & 222 \\
& Total & 422 \\
& Administración & 170 \\
Carrera & Contabilidad & 232 \\
& Marketing & 20 \\
\hline
\end{tabular}

El tamaño de la muestra, de acuerdo a la estadística debió ser de 235. Pero al tener facilidades para acceder a encuestar a más estudiantes se logró aplicar el 
instrumento a 422 estudiantes, logrando de esta manera un trabajo más representativo y consistente con los resultados.

\section{Instrumentos}

Para determinar la relación entre las variables, se utilizó como herramienta de medición, dos instrumentos. Para medir la inteligencia emocional se utilizó el TMMS-24 que contiene las dimensiones: atención emocional (AE), claridad emocional (CE) y reparación emocional (RE); consta de 24 ítems, y se valora con la escala de Likert de cinco puntos: $1=$ En desacuerdo, 2= Algo de acuerdo, 3= Bastante de acuerdo, 4= Muy de acuerdo, $5=$ Totalmente de acuerdo. También se aplicó el cuestionario intención emprendedora que mide las dimensiones: antecedentes personales y familiares (APF), normas sociales (NS), características emprendedoras (CEM), creencias (C), actitud (A) y oportunidades y recursos (OR) y consta de 40 ítems, se valora con una escala de Likert de cinco puntos: $1=$ Totalmente de acuerdo, 2= De acuerdo, 3= Neutro, 4= En desacuerdo, 5= Totalmente en desacuerdo.

\section{Resultados y discusión}

Los resultados fueron elaborados a partir de una análisis descriptivo con el propósito de determinar el nivel de ambas variables. Tambié se realizó un analysis inferencial para determiner el coeficiente de correlación para establecer la prueba de hipótesis.

\section{Análisis descriptivo}

Tabla 1

Nivel de Inteligencia Emocional y sus dimensiones en los estudiantes del ciclo sexto y octavo de la Facultad de Ciencias Empresariales de la Universidad Peruana Unión, resumen de la sede en Lima y sus dos filiales en Tarapoto y Juliaca.

\begin{tabular}{llcc}
\hline & & Frecuencia & Porcentaje \\
\hline & Debe mejorar la inteligencia emocional & 126 & $29.9 \%$ \\
& Adecuada inteligencia emocional & 170 & $40.3 \%$ \\
$\begin{array}{l}\text { Inteligencia } \\
\text { emocional }\end{array}$ & Excelente inteligencia emocional & 126 & $29.9 \%$ \\
& Total & 422 & $100.0 \%$ \\
& Debe mejorar su atención: presta poca & 220 & \\
Atención & atención & & \\
Debe mejorar su atención: & Adecuada atención & 177 & \\
presta demasiada atención & 25 & & $41.9 \%$ \\
& Total & $5.9 \%$ & $100.0 \%$ \\
& & 422 & \\
& Debe mejorar su claridad & & $39.3 \%$ \\
& Adecuada claridad & 166 & $52.1 \%$ \\
& Excelente claridad & 220 & $8.5 \%$ \\
& Total & 36 & $100.0 \%$
\end{tabular}




\begin{tabular}{llcc} 
& Debe mejorar su reparación & 99 & $23.5 \%$ \\
Reparación & Adecuada reparación & 249 & $59.0 \%$ \\
& Excelente reparación & 74 & $17.5 \%$ \\
\hline Total & 422 & $100.0 \%$ \\
\hline
\end{tabular}

La Tabla 1 presenta los resultados para inteligencia emocional: el $29.9 \%$ deben mejorar su inteligencia emocional, el 40.3\% muestran adecuada inteligencia emocional y el $29.9 \%$, una excelente inteligencia emocional. Para la dimensión atención: el $52.1 \%$ \% deben mejorar su atención porque presta poca atención, el $41.9 \%$ muestran adecuada atención y el $5.9 \%$ debe mejorar su atención porque presta demasiada atención. Por otro lado para la dimensión claridad: el $39.3 \%$ debe mejorar su claridad el $52.1 \%$ adecuada claridad y el $8.5 \%$ de los encuestados tienen excelente claridad. Y finalmente para la dimensión reparación el $23.5 \%$ debe mejorar su reparación, el $59.0 \%$ tienen adecuada reparación y el $17.5 \%$ una excelente reparación.

Tabla 2

Nivel de intención emprendedora y sus dimensiones en los estudiantes del ciclo sexto y octavo de la Facultad de Ciencias Empresariales de la Universidad Peruana Unión, resumen de la sede y sus dos filiales.

\begin{tabular}{|c|c|c|c|}
\hline & & Frecuencia & Porcentaje \\
\hline Inicial & 132 & $31.3 \%$ & \\
\hline Intención & En proceso & 164 & $38.9 \%$ \\
\hline \multirow[t]{2}{*}{ emprendedora } & Desarrollada & 126 & $29.9 \%$ \\
\hline & $s / n$ & $s / n$ & $s / n$ \\
\hline Inicial & 171 & $40.5 \%$ & \\
\hline En proceso & 173 & $41.0 \%$ & \\
\hline \multicolumn{4}{|l|}{ Normas } \\
\hline Desarrollada & 78 & $18.5 \%$ & \\
\hline Total & 422 & $100.0 \%$ & \\
\hline Inicial & 144 & $34.1 \%$ & \\
\hline En proceso & 169 & $40.0 \%$ & \\
\hline \multirow[t]{2}{*}{ Desarrollada } & 109 & $25.8 \%$ & \\
\hline & $\mathrm{s} / \mathrm{nl}$ & $s / n$ & $s / n$ \\
\hline Inicial & 147 & $34.8 \%$ & \\
\hline En proceso & 169 & $40.0 \%$ & \\
\hline \multicolumn{4}{|l|}{ Creencias } \\
\hline \multirow[t]{2}{*}{ Desarrollada } & 106 & $25.1 \%$ & \\
\hline & $s / n$ & $s / n$ & $s / n$ \\
\hline Inicial & 140 & $33.2 \%$ & \\
\hline En proceso & 164 & $38.9 \%$ & \\
\hline Actitud & & & \\
\hline
\end{tabular}




\begin{tabular}{llcc} 
Desarrollada & 118 & $28.0 \%$ & \\
& $\mathbf{s} / \mathbf{n}$ & $\mathbf{s} / \mathbf{n}$ & \\
Inicial & 180 & $42.7 \%$ & \\
En proceso & 124 & $29.4 \%$ & $28.0 \%$ \\
Oportunidad & Desarrollada & 118 & $\mathbf{s} / \mathbf{n}$ \\
& $\mathbf{s} / \mathbf{n}$ & $\mathbf{4 2 2}$ & \\
\hline
\end{tabular}

La Tabla 2 muestra los resultados de la variable intención emprendedora: el $38.9 \%$ de los estudiantes encuestados, están en proceso de desarrollar la intención emprendedora, el 31.3\% representa que los estudiantes se encuentran en un nivel inicial y el $29.9 \%$ tienen la intención emprendedora desarrollada. Por otro lado, en la dimensión normas sociales, el $41 \%$ están en proceso de percibir las normas sociales, el $40.5 \%$ indica que se encuentran en un nivel inicial y el $18.5 \%$ ya perciben correctamente las normas sociales. Asimismo para la dimensión características de emprendedores peruanos, el $40 \%$ de los encuestados están en proceso de distinguir las características que identifican a un emprendedor peruano, el $34.1 \%$ se encuentran en un nivel inicial de percibir dichas características, y el $25.8 \%$ representa un nivel desarrollado de percepción de estas características, es decir los encuestados están totalmente de acuerdo con las características. Del mismo modo para la dimensión creencias, los resultados fueron: un $40 \%$ de los encuestados están en proceso de aceptar o no ciertas creencias ya mencionadas en el instrumento; el $34.8 \%$ de los encuestados están en un nivel inicial, queriendo decir que no concuerdan con estas creencias y el otro $25.1 \%$ de encuestados están totalmente de acuerdo a lo mencionado anteriormente, demostrando un nivel desarrollado. También para la variable intención emprendedora, la dimensión actitud se obtuvo los siguientes resultados: el $38.9 \%$ de los encuestados están en proceso de mostrar una actitud emprende- dora, el 33.2\% están en un nivel inicial y el $28 \%$ manifiestan un nivel desarrollado. Finalmente, en la dimensión oportunidad, el $42.7 \%$ de los encuestados percibe oportunidades a un nivel inicial, el $29.4 \%$ se encuentran en proceso y el $28 \%$ un nivel desarrollado.

\section{Prueba de hipótesis}

\section{Hipótesis general}

- Hipótesis nula $\left(\mathrm{H}_{0}\right)$ : La inteligencia emocional no se relaciona significativamente con la intención emprendedora de los estudiantes universitarios en la Facultad de Ciencias Empresariales de la Universidad Peruana Unión, con sede en Lima y sus filiales en Tarapoto y Juliaca, ciclo 2016-II

- Hipótesis alterna $\left(\mathrm{H}_{1}\right)$ : La inteligencia emocional se relaciona significativamente con la intención emprendedora de los estudiantes universitarios en la Facultad de Ciencias Empresariales de la Universidad Peruana Unión, con sede en Lima y sus filiales en Tarapoto y Juliaca, ciclo 2016-II

\section{Regla de decisión}

Si el p valor $>0.05$, se acepta la hipótesis nula.

Si el $p$ valor $<0.05$, se rechaza la hipótesis nula, y se acepta la hipótesis alterna. 
Tabla 3

Relación entre la inteligencia emocional y la intención emprendedora.

\begin{tabular}{|c|c|c|c|}
\hline \multicolumn{4}{|l|}{ Intención emprendedora } \\
\hline & $\begin{array}{l}\text { Rho } \\
\text { de } \\
\text { Spearman }\end{array}$ & p valor & $\mathrm{N}$ \\
\hline Inteligencia emocional &, $247 * \star$ & .000 & 422 \\
\hline
\end{tabular}

En la Tabla 3 se observa la relación entre la inteligencia emocional y la intención emprendedora. Mediante el coeficiente Rho de Spearman se obtuvo 0,247 con un $p$ valor 0,000 , indicando una relación positiva y directa altamente significativa. Es decir que a mayor inteligencia emocional, la intención emprendedora es un rasgo más claro en los estudiantes.

\section{Hipótesis específica 1}

- Hipótesis nula $\left(H_{0}\right)$ : La dimensión atención de las emociones, no se relaciona significativamente con la intención emprendedora de los estudiantes universitarios en la Facultad de Ciencias Empresariales de la Universidad Peruana Unión, con sede en Lima y sus filiales en Tarapoto y Juliaca, ciclo 2016-II.

- Hipótesis alterna $\left(\mathrm{H}_{1}\right)$ : La dimensión atención de las emociones, se relaciona significativamente con la intención emprendedora de los estudiantes universitarios en la Facultad de Ciencias Empresariales de la Universidad Peruana Unión, con sede en Lima y sus filiales en Tarapoto y Juliaca, ciclo 2016-II.

\section{Regla de decisión}

Si el $p$ valor $>0.05$, se acepta la hipótesis nula.
Si el $p$ valor $<0.05$, se rechaza la hipótesis nula, y se acepta la hipótesis alterna.

Tabla 4

Relación entre la dimensión atención de las emociones y la intención emprendedora

\begin{tabular}{llll}
\hline \multicolumn{3}{c}{ Atención } & \\
\hline & $\begin{array}{l}\text { Rho de } \\
\text { Spearman }\end{array}$ & p valor & N \\
\hline $\begin{array}{l}\text { Intención } \\
\text { emprendedora }\end{array}$ &, $182^{\star *}$ & .000 & 422 \\
\hline$* \star$. La correlación es significativa en el nivel 0,01 (bilateral).
\end{tabular}

En la Tabla 4, se observa la relación entre la dimensión atención de las emociones y la variable intención emprendedora. Mediante el coeficiente Rho de Spearman se obtuvo 0.182 con un $p$ valor 0,000 , indicando una relación positiva y directa. Es decir que a mayor atención a las emociones, la intención emprendedora es un rasgo más claro en los estudiantes.

\section{Hipótesis específica 2}

- Hipótesis nula $\left(\mathrm{H}_{0}\right)$ : La dimensión claridad de las emociones, no se relaciona significativamente con la intención emprendedora de los estudiantes universitarios en la $\mathrm{Fa}$ cultad de Ciencias Empresariales de la Universidad Peruana Unión, con sede en Lima y sus filiales en Tarapoto y Juliaca, ciclo 2016-II.

- Hipótesis alterna $\left(\mathrm{H}_{1}\right)$ : La dimensión claridad de las emociones, se relaciona significativamente con la intención emprendedora de los estudiantes universitarios en la Facultad de Ciencias Empresariales de la Universidad Peruana Unión, con sede en Lima y sus filiales en Tarapoto y Juliaca, ciclo 2016-II.

\section{Regla de decisión}

Si el $p$ valor $>0.05$, se acepta la hipótesis nula. 
Si el $p$ valor $<0.05$, se rechaza la hipótesis nula, y se acepta la hipótesis alterna.

Tabla 5

Relación entre la dimensión claridad de las emociones y la intención emprendedora

\begin{tabular}{llll}
\hline & Claridad & & \\
\hline & Rho de Spearman & p valor & $\mathrm{N}$ \\
$\begin{array}{l}\text { Intención em- } \\
\text { prendedora }\end{array}$ &, $164^{\star \star}$ & .000 & 422 \\
\hline
\end{tabular}

**. La correlación es significativa en el nivel 0,01 (bilateral).

En la Tabla 5, se observa la relación entre la dimensión claridad de las emociones y la variable intención emprendedora. Mediante el coeficiente Rho de Spearman se obtuvo 0.164 con $p$ valor 0,000 , indicando una relación positiva y directa, altamente significativa. Es decir que a mayor claridad de las emociones, la intención emprendedora es un rasgo más claro en los estudiantes.

Hipótesis específica 2

- Hipótesis nula $\left(\mathrm{H}_{0}\right)$ : La dimensión reparación de las emociones, no se relaciona significativamente con la intención emprendedora de los estudiantes universitarios en la Facultad de Ciencias Empresariales de la Universidad Peruana Unión con sede en Lima y sus filiales en Tarapoto y Juliaca, ciclo 2016-II.

- Hipótesis alterna $\left(\mathrm{H}_{1}\right.$ : La dimensión reparación de las emociones, se relaciona significativamente con la intención emprendedora de los estudiantes universitarios en la Facultad de Ciencias Empresariales de la Universidad Peruana Unión con sede en Lima y sus filiales en Tarapoto y Juliaca, ciclo 2016-II.

\section{Regla de decisión}

Si el p valor $>0.05$, se acepta la hipótesis nula.
Si el $p$ valor $<0.05$, se rechaza la hipótesis nula, y se acepta la hipótesis alterna.

Tabla 6

Relación entre la dimensión reparación de las emociones y la intención emprendedora

\begin{tabular}{llll}
\hline \multicolumn{3}{l}{ Reparación } & \\
\hline & $\begin{array}{l}\text { Rho de Spear- } \\
\text { man }\end{array}$ & p valor & $N$ \\
Intención emprendedora &, $189 * *$ & .000 & 422 \\
\hline
\end{tabular}

**. La correlación es significativa en el nivel 0,01 (bilateral).

En la Tabla 6, se observa la relación entre la dimensión reparación de las emociones y la variable intención emprendedora. Mediante el coeficiente Rho de Spearman se obtuvo 0.189 con un $p$ valor 0,000 , indicando una relación positiva y directa. Es decir que a mayor reparación de las emociones, la intención emprendedora es un rasgo más claro en los estudiantes.

\section{Conclusiones}

Las conclusiones a partir de los resultados se formulan de acuerdo a los objetivos de la investigación. En primer lugar se presenta los niveles de las variables en estudio $y$, posteriormente, las conclusiones que corresponde a los objetivos generales y específicos.

Los estudiantes del sexto y octavo ciclo de la Facultad de Ciencias Empresariales en la Universidad Peruana Unión con su sede y sus dos filiales, tienen una adecuada inteligencia emocional. Respecto a la primera dimension, la mayoría de los estudiantes deben mejorar su atención sobre sus sentimientos, es decir, prestan poca atención. Respecto a la segunda dimension, la mayoría de los estudiantes tiene una adecuada claridad sobre sus sentimientos. De acuerdo a la tercera dimension, los estudiantes reparan adecuadamente sobre sus sentimientos. 
Del mismo modo, los estudiantes, tiene una intención emprendedora en proceso. Respeco a la dimensiones de norma, características, creencias y actitud también se encuentra en proceso, excepto en la dimension de oportunidad, siendo que la mayoría de los estudiantes en cuanto a posser la característica de saber aprovechar una oportunidad de negocios se encuentra en un incipiente nivel.

Por último, de acuerdo al objetivo principal del trabajo se concluye que los niveles de intención emprendedora e inteligencia emocional de los estudiantes del ciclo sexto y octavo de la Facultad de Ciencias Empresariales en la Universidad Peruana Unión y sus tres filiales, presentan una correlación positiva y significativa, demostrándose que a mayor nivel de inteligencia emocional, los estudiantes unionistas tienen mayor intención de emprender.

También se concluye que las dimensiones de inteligencia emocional, presentan una correlación positiva y significativa con la variable intención emprendedora. Es decir que a mayor atención de las emociones, claridad de las emociones y reparación o regulación de las emociones, mayor intención de emprender, tendrá el estudiante unionista.

\section{Recomendaciones}

Se debe implementar un ecosistema emprendedor, reactivando una incuba- dora de empresas, en la facultad, que permita realizar talleres de capacitación, consultoría, asistencia técnica, canalizar financiamiento para las iniciativas emprendedoras de los estudiantes y acompañarlos hasta que el negocio esté bien implementado. También promover la particpación en concursos, enferias e implimentar una clínica de negocios, para generar experiencias propias, afrontando riesgos reales. Así, la Facultad de Ciencias Empresariales estaría cumpliendo con la ley N³0220 (Ley Universitaria), aprobada el 03/07/2014 que promueve un ambiente de ecosistema emprendedor, señalando en el capítulo $\mathrm{VI}$, articulo 52, la responsabilidad de desarrollar la iniciativa de los estudiantes para la creación de pequeñas y microempresas de propiedad de los estudiantes, brindando asesoría o facilidades en el uso de los equipos e instalaciones de la institución.

Se recomienda tomar en cuenta las horas de tutoría para desarrollar talleres de inteligencia emocional con un enfoque empresarial, resolviendo casos hipotéticos para tomar decisiones bajo presión; estimulando y fortaleciendo de esta manera sus capacidades emprendedoras e innovadoras. Y con el objetivo de fomentar ideas, de superar sus miedos, desconfianzas y temores al fracaso, siendo así más proactivos y llevándolos al éxito empresarial. 


\section{Referencias}

Abrego, R., González, K., y Lullo, R. (2012). Elaboración de un diseño instruccional para el desarrollo de habilidades en el manejo de conflictos y negociaciones dirigida al área de mercadeo y ventas en la industria farmacéutica Roka C.A., utilizando la inteligencia emocional (tesis de licenciatura). Universidad Central de Venezuela. Caracas, Venezuela.

Acuña, C. (2014). Aplicación de la metodología CEFE como herramienta para el desarrollo de las capacidades empresariales personales a los estudiantes de la Facultad de Ciencias Empresariales de la UPeU-filial Tarapoto. Valor Agregado Revista de Investigación de Administración, 1(1), 0920.

Alonso, M., y Galve, C. (2008). El emprendedor y la empresa: una revisión teórica de los determinantes a su constitución. Revista Acciones e Investigaciones Sociales, (26), 5-44.

Alvaro, M., Mendiri, M., y Paz, A. (2003). Relación entre el perfil de los emprendedores venezolanos en edades comprendidas entre 18 y 42 años y el éxito (tesis de bachiIler). Universidad Metropolitana. Caracas.

Bautista, N. (2014). Diseño de una propuesta para fortalecer las habilidades de emprendimiento productivo en los estudiantes de la Universidad Tecnológica de la Mixteca (tesis de licenciatura). Universidad Tecnológica de la Mixteca. Huajapan de León, México.

Bollón, A. (2010). Cerindetec I+D+i. Recuperado el 21 de Abril de 2016, de http://www. cerindetec.com/resources/Inteligencia+Emocional.pdf

Cabrera, M. (2011). Inteligencia emocional y rendimiento académico de los alumnos del nivel secundario de una institución educativa de la región Callao (tesis de maestría). Universidad San Ignacio de Loyola. Lima, Lima, Perú.

Celaya, R. (2013). Rumbo a la cumbre 2 (Primera ed.). México: Emprende7joven.

Cooper, R., y Sawaf, A. (1998). La inteligencia emocional aplicada al liderazgo y a las organizaciones. Bogotá, Colombia: Editorial Norma.

Del Fabro, A. (03 de Mayo de 2013). ¿Por qué el coeficiente emocional es más importante que el coeficiente intelectual? Recuperado el 29 de Abril de 2016, de PSYCIENCIA: http://www.psyciencia. com/2013/03/por-que-el-coeficienteemocional-es-mas-importante-que-el-coeficiente-intelectual/

Diez, A. (2011). Coeficiente emocional vs. intelectual. Recuperado el 29 de Abril de 2016, de Management sin corbata. Recuperado de http://www.managementsincorbata.com/coeficienteemocional-vs-intelectual/

Extremera, N., y Fernández, P. (2003). La inteligencia emocional en el contexto educativo: hallazgos científicos de sus efectos en el aula. Revista de Educación, (233), 97-116.

Extremera, N., y Fernández, P. (2004). Inteligencia emocional, calidad de las relaciones interpersonales y empatía en estudiantes universitarios. Red de Revistas Científicas de America Latina, el Caribe, España y Portugal, 15(2), 117-137.

Ferrando, M. (2006). Creatividad e inteligencia emocional: un estudio empírico en alumnos con altas habilidades (tesis doctoral). Universidad de Murcia. España. 
Fernández, P., y Ruiz, D. (2008). La inteligencia emocional en la educación. Revista Electrónica de Investigación Psicoeducativa, 4(15), 421-436.

Fernández, A. (2010). Inteligencia emocional como variable predictora de adaptación psicosocial en estudiantes de la comunidad de Madrid (tesis doctoral). Universidad Autónoma de Madrid. España.

Fretes, P., Venturi, J. y Villalba, E. (2016). Estudio del perfil emprendedor de los empresarios de Pilar, según la escuela comportamentalista de David McClelland. Revista Electrónica Científica de la UNP. Universidad Nacional de Pilar - UNP. Recuperado a partir de http://www.ebah. com.br/content/ABAAAfkZUAA/estudio-del-perfil-emprendedor-los-empresarios-pilar-segun-la-escuela-comportamentalista-david-mcclelland.

Gómez, J., Mira, I., Martínez, J., y Estrada, M. (2012). Actividad emprendedora en la comunidad Valenciana. Universidad Miguel Hernández. Valencia. España

Hernández, M., Cabellero, M., y Monroy, J. A. (2013). Emprendedurismo y propensión a la creación de negocios de los estudiantes de la FCA de la UAEMEX. XVI Congreso internacional sobre innovaciones en docencia e investigación en ciencias económico administrativas, 6-7.

Hidalgo, G., Kamiya, M., y Reyes, M. (2014). Emprendimientos dinámicos en América Latina. Avances en prácticas y políticas. México: Serie Políticas Públicas y Transformación Productiva. Banco de Desarrollo de América Latina.

Kelley, D., Singer, S., y Herrington, M. (2016). 2015/16 Global Report. London: GEMconsortium.
Maestres M., (2006). La inteligencia emocional y el éxito en la gerencia. Debates IESA, 11(2), 8-10.

Manrique, F. (2012). Inteligencia Emocional y rendimiento académico en estudiantes del $V$ ciclo primaria de una institución educativa de Ventanilla-Callao. Lima, Perú: Universidad San Ignacio de Loyola.

Martín de Benito, M. (2013). Análisis de un modelo estructural de inteligencia emocional y motivación autodeterminado en el deporte (tesis doctoral). Universidad de Valencia. España.

Nueno, P. (2007). Cartas a un joven emprendedor. España: Editorial Crítica.

Numancia, C. (2013). Estilos de aprendizaje en el desarrolo de capacidades de emprendimiento en estudiantes de secundaria (tesis de maestría). Universidad San Martín de Porres. Lima, Perú.

Pittaluga, C. (2012). La inteligencia emocional en las escuelas degerencia. Debates IESA, 17(4), 46-49.

Puchol, L. (2005). El libro del emprendedor (Segunda ed.). España: Ediciones Díaz de Santos.

Quintillán, I. (2005). Liderazgo e inteligencia emocional: la formación y el perfil de los estudiantes del último año de la licenciatura en dirección de empresas. Revista Electrónica de la Facultad de Ciencias Empresariales de la Universidad Católica del Uruguay, 1-17.

Reátegui, M. (11 de Mayo de 2015). Perú, quinta economia con mayor emprendimiento en el mundo. RPP Noticias.

Salazar, C. (13 de Octubre de 2016). Sala de prensa, Universidad Catolica San Pablo. 
Obtenido de https://ucsp.edu.pe/saladeprensa/informa/la-estructuraempresarial-de-peru-no-ayuda-al-desarrollo/

Salguero, J., Fernández, P., Ruiz, D., CastiIlo, R., y Palomera, R. (2015). Inteligencia emocional y ajuste psicosocial en la adolescencia: el papel de la percepción emocional. European Journal of Education and Psychology, 4(2), 143-152.

Silva, E. G. (2013). Emprendedor. Hacia un emprendimiento sostenible. México: Editorial Alfaomega.

Singer, S., Amorós, J., y Moska, D. (2014). Global Entrepreneurship Monitor 2014 - Global Report. London: GEMConsortium.
Soto, F. E., De la Torre, A., y Piñón, O. (2014). Medición de inteligencia emocional y clima organizacional en relación a factores de riesgo psicosocial en empresa creadora de almacenes de la ciudad de Chihuahua. XVI Congreso Internacional Sobre Innovaciones en Docencia e Investigación en Ciencias Económico Administrativas. Chihuahua, México: Universidad Autónoma de Chihuahua.

Villarán, F. (2004). Emprende empresa: así comenzaron los empresarios de éxito. Lima: Ministerio de Trabajo y Promoción del Empleo.

Zuñiga, Q. (19 de Mayo de 2015). Las pymes y la economía peruana. La República, pág. 14. 